\section{ВЫПУКЛОСТЬ ОГРАНИЧЕННЫХ ЧЕБЫШЁВСКИХ МНОЖЕСТВ В КОНЕЧНОМЕРНЫХ ПРОСТРАНСТВАХ С НЕСИММЕТРИЧНОЙ НОРМОЙ}

\section{А. Р. Алимов}

Кандидат фризико-математических наук, научный сотрудник лаборатории вычислительных методов механико-математического фракультета, Московский государственный университет им. М. В. Ломоносова, alexey.alimov@gmail.com

Известная характеризация Царькова конечномерных банаховых пространств, в которых всякое ограниченное чебышёвское множество (ограниченное $P$-ацикличное множество) выпукло, обобщается на несимметричный случай.

Ключевые слова: ограниченное чебышёвское множество, выпуклость, несимметричная норма.

Множество $M$ из пространства $X$ с фиксированной функцией расстояния $\rho: X \times X \rightarrow \mathbb{R}_{+}$называется чебышёвским множеством, если для каждой точки $x \in X$ множество $P_{M} x:=\inf _{y \in M} \rho(x, y)$ ее ближайших точек из $M$ состоит из одной точки. Исторически первыми интересными примерами чебышёвских множеств служили подпространства многочленов, тригонометрических полиномов и множество дробно-рациональных функций в $C[0,1]$. Единственность наилучшего приближения такими множествами была отмечена в XIX веке П. Л. Чебышёвым. Множества со свойством существования и единственности элемента наилучшего приближения были названы Ефимовым и Стечкиным [1] чебышёвскими в честь Чебышёва. В данной работе рассматривается вопрос выпуклости ограниченных чебышёвских множеств в конечномерных несимметрично нормированных пространствах.

Далее всегда предполагается, что $M \neq \varnothing, X-$ конечномерное линейное действительное пространство, в качестве функции расстояния $\rho$ в котором служит норма или несимметричная норма, определяемая ниже.

В этой работе мы изучаем взаимосвязь между выпуклостью чебышёвских множеств в $X$ и геометрическими свойствами пространства $X$. На конечномерные пространства с несимметричной нормой обобщается следующая характеризация Царькова [2], которую мы кратко сформулируем следующим образом: в конечномерном линейном нормированном пространстве $X$ всякое ограниченное чебьшёвское множество выпукло тогда и только тогда, когда множество экстремальных точек единичной сферы $S^{*}$ сопряженного пространства плотно в ней.

Напомним [3], что несимметричной нормой $\|\cdot\|$ на действительном линейном пространстве $X$ называется функционал Минковского некоторого фиксированного выпуклого ограниченно поглощающего
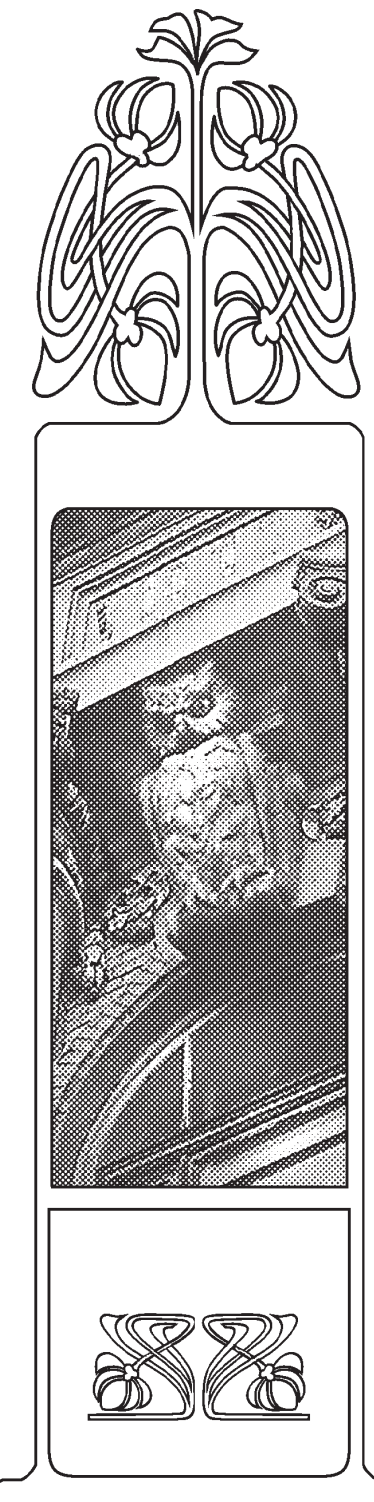

НАУЧНЫЙ
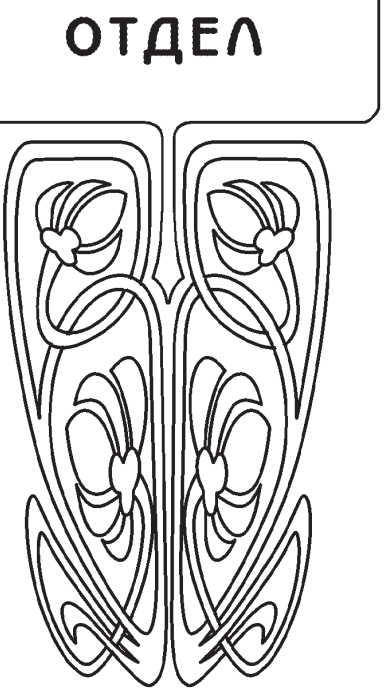
множества $B$ (единичного шара), содержащего начало координат в своем ядре. Именно для $x, y \in X$, $x \neq 0$ определяется

$$
\|x\|:=\sup \{\sigma \geqslant 0 \mid x \notin \sigma B\}, \quad\|0\|=0 .
$$

Несимметричные нормы возникли впервые у Г. Минковского («функционал Минковского»), в бесконечномерный анализ они были привнесены М. Г. Крейном. Термин несимметричная норма был предложен Крейном [4, с. 197] в 1938 г.

Несимметричная норма удовлетворяет всем аксиомам нормы, исключая аксиому симметрии; в общем случае $\|x-y\| \neq\|y-x\|$. Неравенство треугольника выполнено в следующем виде:

$$
\|x-y\| \leqslant\|x-z\|+\|z-y\|
$$

для любых $x, y, z \in X$.

В последние годы активно развивается тематика, связанная несимметричными нормами и односторонними приближениями (С. Кобзаш (S. Cobzaş) [3], Х.-П. А. Кюнци (Н.-P. A. Künzi), С. Ромагуера (S. Romaguera), К. Алегре (С. Alegre), Э. А. Санчес Перез (Е. А. Sánchez Pérez), П. А. Бородин [5] и др.). Наиболее полно обзор результатов, относящихся к общим вопросам функционального анализа в несимметрично нормированных пространствах приведен в недавно вышедшей книге С. Кобзаша [3].

Основным результатом настоящей работы является обобщение характеризации Царькова на несимметричный случай. Попутно мы подчеркиваем роль инструментария геометрической топологии, в очередной раз продемонстрировавшего свою применимость в решении задач геометрической теории приближений.

Проблема выпуклости чебышёвских множеств в конкретных и абстрактных банаховых пространствах рассматривалась многими авторами (см., например, В.И. Бердышев [6], А. Брондстед (A. Brøndsted) [7], А. Л. Браун (А. L. Brown) [8, 9], Л. П. Власов [10], В. С. Балаганский и Л. П. Власов [11], И. Г. Царьков $[2,12]$, А. Р. Алимов $[13,14])$. Коротко напомним результаты, относящиеся к конечномерному случаю.

Точка $s$ единичной сферы $S$ несимметрично нормированного пространства $X$ называется точкой гладкости (гладкой точкой), если в ней опорная гиперплоскость к $S$ единственна; точка $s-\partial о-$ стижимая точка сферы $S$ (или единичного шара $B$ ), если существует опорная к $B$ гиперплоскость, пересекающая $B$ в точности по точке $s$. Множество экстремальных (крайних), достижимых (выставленных) и гладких точек единичной сферы $S$ будет обозначаться соответственно посредством $\operatorname{ext} S$, $\exp S$ и $\operatorname{sm} S$. Пространство $X$ называется гладким, если $\operatorname{sm} S=S$.

Изучение геометрии чебышёвских множеств в линейных нормированных пространствах было начато Л. Бунтом (L. N. H. Bunt) [15], который доказал, что в строго выпуклых конечномерных пространствах с модулем гладкости второго порядка (в частности, в евклидовых) любое чебышёвское множество выпукло, причем в двумерном случае он показал, что от условия строгой выпуклости можно избавиться. Т. С. Моцкин и Х. Манн (см. $[10,11,13,16])$ показали, что в двумерном случае гладкость пространства - необходимое и достаточное условие для выпуклости любого чебышёвского множества в этом пространстве.

Следующее серьёзное продвижение в исследовании чебышёвских множеств связано с работами В. Кли (V. L. Klee), Н. В. Ефимова и С. Б. Стечкина. В своих ранних работах Кли $[17,18]$ обобщил теоремы Бунта и Моцкина, показав, что каждое чебышёвское множество является выпуклым в любом конечномерном гладком пространстве. Ефимов и Стечкин (см. $[10,13,16])$ доказали, что в конечномерном банаховом пространстве $X$ класс всех чебышёвских множеств совпадает с классом всех выпуклых замкнутых множеств тогда и только тогда, когда $X$ строго выпукло и гладко.

А. Брондстед [7] охарактеризовал трехмерные несимметрично нормированные пространства, в которых всякое чебышёвское множество выпукло (и отметил, что результат Моцкина - Манна верен также и для двумерных несимметрично нормированных пространств). Независимо в то же самое время В. И. Бердышев [6] получил ту же характеризацию, но лишь для нормированного случая. В [9] Браун получил характеризацию четырехмерных линейных нормированных пространств, в которых всякое чебышёвское множество выпукло, позднее автор данной статьи [14] перенес характеризацию Брауна на несимметричный случай.

Сформулируем результаты Бердышева - Брондстеда - Брауна - Алимова в виде следующей теоремы (см. $[6,7,9,13,14])$. 
Теорема. Пусть X - несимметрично нормированное пространство размерности 3 или 4. Каждое чебышёвское множество в X выпукло, если и только если каждая достижимая точка единичного шара пространства $X$ является точкой гладкости.

В дополнение к упомянутой характеризации Бердышев для $n=3$ и Брондстед для всех натуральных $n \geqslant 3$ построили примеры негладких пространств, в которых всякое чебышёвское множество выпукло.

Вопрос о характеризации $n$-мерных пространств, в которых всякое чебышёвское множество выпукло, открыт для $n \geqslant 5$ (подробнее см. [9, 11, 13]).

Удивительно, но решение задачи о выпуклости чебышёвских множеств принципиально отличается в зависимости от того, рассматриваются ли ограниченные или неограниченные множества.

Ниже мы рассматриваем вопрос о характеризации пространств (в том числе несимметрично нормированных), в которых всякое ограниченное чебышёвское множество выпукло.

В 1958 г. Н. В. Ефимов и С. Б. Стечкин [1] сформулировали следующую задачу: охарактеризовать конечномерные линейные нормированные пространства, в которых всякое ограниченное чебышёвское множество выпукло. Полный ответ на этот вопрос был получен Царьковым в [2] (см. также и работу Брауна [8], в которой подробно разбирается доказательство Царькова и исследуются возникающие при этом сопутствующие задачи).

Рассматриваемая в работе задача для несимметрично нормированных пространств была поставлена в 1990-х гг. С. Б. Стечкиным, который был активным сторонником рассмотрения задач геометрической теории приближений в несимметричном случае.

Для того чтобы сформулировать результат Царькова о выпуклости ограниченных чебышёвских множеств, нам потребуется несколько определений и фактов.

Далее $X-$ конечномерное несимметрично нормированное пространство, $\|\cdot\|-$ несимметричная норма на $X$. Для $x \in X$ и $r>0$ положим:

$S(x, r):=\{y \in X \mid\|x-y\|=r\}-$ сфера,

$B(x, r):=\{y \in X \mid\|x-y\| \leqslant r\}-$ замкнутый шар,

$\stackrel{\circ}{B}(x, r):=\{y \in X \mid\|x-y\|<r\}-$ открытый шар

соответственно с центром $x$ и радиусом $r$. Единичный шар $B(0,1)$ обозначается посредством $B, S-$ единичная сфера. Сопряженное пространство к $X$ обозначается $X^{*}$; при этом естественная несимметричная норма на $X^{*}$ для $f, g \in X^{*}$ определяется как $d(0, f):=\max _{x \in B} f(x), d(f, g):=d(0, g-f)$. Для конечномерного (или метризуемого) $X$ сопряженное является линейным пространством, в бесконечномерном неметризуемом случае в качестве сопряженного естественно возникают конусы. Единичная сфера пространства $X^{*}$ относительно введенной несимметричной нормы $d$ обозначается посредством $S^{*}$, а единичный шар $-B^{*}$. Естественная топология на $X$ порождается базой $\{\stackrel{\circ}{B}(x, r)\}$; если $\operatorname{dim} X=n<\infty$, то эта топология совпадает с обычной евклидовой топологией на $\mathbb{R}^{n}$.

Напомним еще несколько определений.

Для $x \in S$ посредством $V(x)$ обозначим объединение 0 и всех прямых линий $\ell$ таких, что

$$
\ell \cap \operatorname{cl}\{\lambda(B-x) \mid \lambda \geqslant 0\}=\{0\} .
$$

Буква «V» означает первую букву латинского «visibilitas» («видимость») - таким образом, множество $x+V(x)$ состоит из всех точек вне единичного шара $B$, из которых можно «напрямую» (в строгом смысле) увидеть точку $x$ на поверхности $S$ шара $B$ (см. [8]).

Подмножество $R \subset S$ называется хребтом (ridge) [8] сферы $S$, если оно связно и найдутся подпространство $L \subset X, \operatorname{dim} L \geqslant 0$, и открытое в $R$ подмножество $W \subset R$ такие, что

1) $L \backslash\{0\} \subset \operatorname{int} \bigcap_{x \in R} V(x)$;

2) $W$ гомеоморфно $\mathbb{R}^{(\operatorname{dim} X-1-\operatorname{dim} L)}$.

Хребет $R$ называется простым хребтом, если существует открытое подмножество $U$ из $S^{*}$ такое, что $R=\cup\left\{f^{-1}(1) \cap S \mid f \in U\right\}$.

Примеры и свойства хребтов [8]:

1. Связное открытое в $S$ подмножество $R \subset S$ является хребтом размерности $(\operatorname{dim} X-1)($ здесь $L=\{0\})$. Такой хребет называется купольным.

2. Хребет размерности 0 - это точка, называемая «пиком». 
3. Пусть $X-$ пространство $\left(\mathbb{R}^{3},\|\cdot\|_{\infty}\right)$. Вершины единичного куба являются простыми пиками; интервал, содержащийся в ребре куба, - это хребет, но не простой хребет; ребро куба - простой хребет.

Следующий результат был доказан в [8] без упоминания пространств с несимметричной сферой. Тем не менее его можно перенести безо всякого изменения на случай несимметрично нормированных пространств.

Предложение А. Пусть $R$ - хребет на сфере $S$, а $L$ - подпространство, фигурирующее 8 определении хребта $F$. Далее, пусть $\pi: X \rightarrow X / L-$ каноническое фактор-отображение. Тогда внутренность $\pi(R)$ в $S(X / L)$ непуста, а топологическая размерность $R$ равна $\operatorname{dim} X-1-\operatorname{dim} L$.

Из предложения А следует, что выражение «хребет размерности $k »$ корректно.

Следуя [8], определим

$$
\begin{array}{ll}
\Psi_{*}(x)=\left\{f \in S^{*} \mid f(x)=1\right\}, & x \in X, \\
\Psi(f)=\{x \in S \mid f(x)=1\}, & f \in S^{*} .
\end{array}
$$

Если $U \subset S^{*}$, то $\Psi(U)$ обозначает множество $\cup\{\Psi(f) \mid f \in U\}$. Таким образом, $\Psi_{*}(x)-$ достижимая грань сферы $S^{*}$, определяемая посредством $x \in S$, и $\Psi(f)$ - достижимая грань сферы $S$, определяемая посредством $f \in S^{*}$. В наших терминах точка $x \in S$ есть точка гладкости, тогда и только тогда, когда $\Psi_{*}(x)$ состоит из одной точки.

Коническое множество [2] определяется следующим образом: подмножество $C$ сферы $S$ называется коническим множеством, если найдутся одномерное подпространство $L$ пространства $X$ и открытое подмножество $U_{0}$ сферы $S^{*}$ такие, что

1) $L \backslash\{0\} \subset \operatorname{int}\{\cap V(x) \mid x \in C\}$;

2) $\Psi\left(U_{0}\right) \subset C$.

Из этого определения немедленно следует, что

простой хребет размерности, меньшей чем $\operatorname{dim} X-1$, является

коническим множеством.

Один из основных результатов работы Царькова [2] состоит в том, что если сфера $S$ линейного нормированного пространства $X$ содержит коническое множество, то в $X$ имеется невыпуклое ограниченное чебышёвское множество. Аналогичный результат верен и в несимметричном случае.

Теория гомологий (когомологий) связывает с каждым топологическим пространством $X$ последовательности абелевых групп $H_{k}(X), k=0,1,2, \ldots$ (группы гомологий) и $H^{k}(X), k=0,1,2, \ldots$ (группы когомологий), которые являются гомотопическими инвариантами пространства: если два пространства гомотопически эквивалентны, то и соответствующие группы гомологий изоморфны.

Пусть $A-$ произвольная нетривиальная абелева группа. Пространство (все пространства предполагаются метризуемыми) называется ацикличным, если его группа чеховских когомологий с коэффициентами из $A$ тривиальна (не имеет циклов, за исключением границы). Таким образом, определение ацикличности зависит от выбранной группы коэффициентов. В случае если гомология (когомология) имеет компактный носитель и коэффициенты группы гомологий (когомологий) лежат в поле, то понятия гомологической и когомологической ацикличности совпадают.

Далее, если не оговорено противное, ацикличность будет пониматься относительно чеховских когомологий с коэффициентами в произвольной абелевой группе.

Множество $M$ называется $P$-ацикличным, если для всякого $x \in X$ множество $P_{M} x$ ближайших точек из $M$ к $x$ непусто и ациклично. Множество $B$-ациклично, если его пересечение с любым шаром ациклично.

Для наших целей важно отметить $[8,9]$, что если $M-P$-ацикличное аппроксимативно компактное подмножество банахова пространства и если пересечение $M$ с некоторым шаром $B$ компактно, то $M \cap B$ ациклично; в частности, в банаховом пространстве для ограниченно компактных множеств класс $P$-ацикличных множеств совпадает с классом $B$-ацикличных множеств [8, лемма 5.3].

Понятно, что всякое чебышёвское множество $P$-ациклично. Также отметим, что ограниченно компактное $P$-ацикличное подмножество банахова пространства всегда является солнцем (см. $[10$, теорема 4.4]).

В следующей основной теореме характеризация Царькова распространяется на конечномерные пространства с несимметричной нормой. 
Теорема 1. Пусть $X-$ конечномерное несимметрично нормированное пространство. Тогда следующие условия эквиваленты:

a) всякое ограниченное чебышёвское множество в X выпукло;

б) всякое ограниченное $P$-ацикличное множество в $X$ выпукло;

в) всякое ограниченное $B$-ацикличное множество в $X$ выпукло;

2) $\overline{\exp } S^{*}=S^{*}$;

d) $\overline{\operatorname{ext}} S^{*}=S^{*}$

е) сфера $S$ не содержит конических подмножеств;

ж) сфера $S$ не содержит простых хребтов размерности $<\operatorname{dim} X-1$.

В симметричном случае эквивалентность е) другим условиям в теореме 1 , а также равносильность б) $\Leftrightarrow$ в) была установлена Брауном [8].

Мы докажем б) $\Leftrightarrow$ в) $\Rightarrow$ а) $\Rightarrow$ ж) $\Rightarrow$ е) $\Rightarrow$ д) $\Leftrightarrow$ г) $\Rightarrow$ б).

г) $\Leftrightarrow$ д) гарантируется классической теоремой Страшевича, согласно которой множество достижимых точек конечномерного выпуклого компакта образует плотное подмножество во множестве его крайних точек.

Импликация ж) $\Rightarrow$ е) следует из $(2)$ : простой хребет размерности $<\operatorname{dim} X-1$ является коническим множеством.

а) $\Rightarrow$ ж) вытекает из следующего результата Царькова [2, лемма 5].

Лемма А. Пусть $X-$ конечномерное нормированное пространство. Предположим, что на единичной сфере $S$ найдется коническое множество. Тогда существует невыпуклое ограниченное чебышёвское множество в $X$.

На случай несимметрично нормированных пространств доказательство леммы А переносится без изменений.

Импликация г) $\Rightarrow$ б) основывается на следующих утверждениях, из которых лемма В (см. $[2,8])$ достаточно интуитивно ясна: полукруг не выпуклый, следовательно, существует гиперплоскость, «отсекающая кусочки» от концов полукруга; полусфера также не выпукла, и поэтому возможно отсечь от нее гиперплоскостью обод. Как и выше, симметричность в лемме В не по существу.

Лемма В. Пусть $X-$ конечномерное несимметрично нормированное пространство и $M \subset X$. Если $M$ - ограничено, замкнуто, невыпукло и $X \backslash M$ связно, то тогда существует открытое полупространство $H$ и множество $\Sigma \subset H \cap M$ такие, что

1) $\Sigma$ является относительной границей некоторого выпуклого множества и, следовательно, $\Sigma$ гомеоморфно сфере;

2) $\Sigma$ является ретрактом $H \cap M$.

Напомним, что по определению подмножество $A \subset C$ называется ретрактом множества $C$, если существует непрерывное отображение $r: C \rightarrow A$, называемое ретракцией, такое, что $\left.r\right|_{A}=\left.1\right|_{A}$, т. е. тождественное отображение $1_{A}$ допускает непрерывное продолжение на все пространство $C$. Следующий результат хорошо известен. Пусть $M-$ чебышёвское множество с непрерывной метрической проекцией, а $B-$ замкнутый шар, $B \cap M \neq \varnothing$. Тогда $M \cap B-$ ретракт шара $B$ (т. е. $M-B$-ретракт). Давно известно, что конечномерная сфера не является ретрактом замкнутого шара, границей которого она служит (в бесконечномерном случае это уже не так). Аналогом теоремы об отсутствии ретракций для 2-значных ретракций служит лемма 2 , которая нам понадобится при перенесении утверждения о чебышёвских множествах на случай $P$ - и $B$-ацикличных множеств.

Через $\exp _{2} A$ обозначается пространство всех, не более чем двухточечных подмножеств $A$ с топологией Вьеториса. Пусть $A \subset C$. Непрерывное (т.е. полунепрерывное сверху и снизу) отображение $r: C \rightarrow \exp _{2}(A)$ называется 2-значной $\left(\exp _{2}\right.$-значной $)$ ретракцией $C$ на $A$, если $\left.r\right|_{A}=\mathrm{id}$.

Следующие два результата были сообщены автору Е. В. Щепиным и А. Н. Дранишниковым.

Лемма 1. Пусть $X$ - конечномерное несимметрично нормированное пространство и пусть $M \subset X-B$-ацикличный компакт. Тогда существует 2-значная ретракция $B(x, r)$ на $B(x, r) \cap M$.

Напомним, что компакт $Y$ называется клеточноподобным (имеющим шейп точки), если существует ANR $Z$ и вложение $i: Y \rightarrow Z$ такое, что образ $i(Y)$ стягиваем в любой своей окрестности $U \subset Z$. Согласно известной характеризации Д. Химана (D. Hyman) компакт клеточноподобен если и только если он гомеоморфен пересечению счетной убывающей последовательности абсолютных ретрактов (или стягиваемых компактов), т. е. является $R_{\delta}$-множеством в смысле Ароншайна. 
Для доказательства леммы 1 сначала заметим, что что симметрический квадрат (в смысле Борсука и Улама) ацикличного компакта клеточноподобен) [19]. Далее, согласно классической теореме А. Дольда (A. Dold) [20, Theorem 5.11], для полиэдров целочисленные гомологии пространства $\exp _{2} Y$ полностью определяется гомологиями пространства $Y$, т. е. если $H_{i}(Y)=H_{i}\left(Y^{\prime}\right)$ при всех $i \leqslant n$, то тогда $H_{i}\left(\exp _{2} Y\right)=H_{i}\left(\exp _{2} Y^{\prime}\right), i \leqslant n$. Окончательно, отсюда следует, что при вложении в симметрический квадрат ацикличного компакта стягиваемость диагонали эквивалентна существованию $\exp _{2}$-значной ретракции, иными словами, отображение диагонали продолжается до отображения диска.

Лемма 2. Не существует 2-значной ретракции конечномерного п-шара на сферу.

Лемма 2 есть следствие теоремы Дольда-Тома (A. Dold, R. Thom) (см. [19, § 3.E; 21]), согласно которой бесконечное симметрическое произведение $\mathrm{SP}^{\infty}\left(S^{n}\right) n$-сферы является комплексом Эйленберга-Маклейна $K(\mathbb{Z}, n)$ (т. е. $\mathrm{CW}$-комплексом $K$, у которого гомотопические группы $\pi_{i}(K)$ тривиальны при $i \neq n$ и $\left.\pi_{n}(K)=G\right)$, а вложение $S^{n} \rightarrow \mathrm{SP}^{\infty}$ гомотопически нетривиально. Комплекс $K(\mathbb{Z}, n)$ можно понимать как $\mathrm{CW}$-комплекс, получающийся из $n$-мерной сферы $S^{n}$ добавлением клеток размерности $\geqslant n+2$. Как следствие, вложение $S^{n} \subset \mathrm{SP}^{2}\left(S^{n}\right)$ в симметрический квадрат гомотопически нетривиально. Поэтому его нельзя продолжить на шар $B^{n+1}$, что показывает отсутствие 2-значной (непрерывной) ретракции. С другой стороны, отметим, что 3-значная ретракция шара на границу всегда существует (Дранишников [22]).

Теперь импликация г) $\Rightarrow б)$ может быть завершена при помощи следующих рассуждений. Предположим, что $\overline{\exp } S^{*}=S^{*}$, и существует невыпуклое ограниченное $P$-ацикличное множество $M$. Пусть $H$ и $\Sigma$ выбраны как в лемме В (лемма применима, так как дополнение ко множеству $M$ связно: действительно, будучи $P$-ацикличным $M$ является солнцем по известной теореме Власова [10, теорема 4.4], а дополнение к ограниченному солнцу всегда связно [23]). Полупространство $H$ определяется посредством некоторого функционала $f \in S^{*}$. Слегка изменяя $H$, мы не меняем ситуации, так что мы можем предположить, что $f \in \exp S^{*}$. Тогда если гиперплоскость bd $H$ является опорной к некоторому шару, то касание происходит по гладкой точке. Соответственно можно выбрать замкнутый шар $B_{R}$ достаточно большого радиуса, таким образом, что $\Sigma \subset\left(B_{R} \cap M\right) \subset(H \cap M)$. По лемме 1 множество $\Sigma$ является ретрактом $B \cap M$, а множество $B \cap M-2$-ретрактом $B$, но это невозможно, так как по лемме 2 сфера не может быть 2 -ретрактом шара. Это противоречие доказывает импликацию г) $\Rightarrow$ б).

Доказательство импликации е) $\Rightarrow$ д) не меняется при переходе с симметричного случая на несимметричный.

Для доказательства б) $\Leftrightarrow$ в) воспользуемся следующим утверждением, доказанным Брауном [8] для случая общих линейных нормированных пространств.

Лемма 3. Пусть $M$ - замкнутое подмножество конечномерного несимметрично нормированного пространства $X$. Если $B=B(z, R)$ - замкнутый шар и $B \cap M \neq \varnothing$, то существует полунепрерывная сверху многозначная ретракция $\Phi: B \rightarrow B \cap M$ такая, что для каждого $z \in B$ множество $\Phi(z)$ совпадает с $P_{M}\left(z^{\prime}\right)$ для некоторого $z^{\prime} \in B$.

Доказательство. Мы используем схему рассуждений Брауна. Пусть $B=B\left(z_{0}, R\right)$. Для $z \in B$ положим $k(z)=\sup \left\{k \in[0,1] \mid \rho\left((1-k) z+k z_{0}, M\right)=k R\right\}$. Ясно, что $\rho\left(z_{0}, M\right) \leqslant R$ и $\rho(z, M) \geqslant 0$. Отсюда вследствие непрерывности функции расстояния точка $k(z)$ определена корректно, при этом $\rho\left((1-k(z) z)+k(z) z_{0}, M\right)=k(z) R$. Определим $\Phi$ следующим образом:

$$
\Phi(z)=P_{M}\left((1-k(z)) z+k(z) z_{0}\right) .
$$

Понятно, что имеет место следующее включение шаров:

$$
B\left((1-k) z+k z_{0}, k R\right) \subset B\left(\left(1-k^{\prime}\right) z+k^{\prime} z_{0}, k^{\prime} R\right), \quad 0 \leqslant k \leqslant k^{\prime} \leqslant 1
$$

(при этом мы воспользовались тем, что $B(x, r) \subset B\left(x^{\prime}, r^{\prime}\right)$, если и только если $\left\|x-x^{\prime}\right\| \leqslant r^{\prime}-r$, что верно в произвольных несимметрично нормированных пространствах).

Имеем: $\Phi(z)=B\left((1-k(z)) z+k(z) z_{0}, k(z) R\right) \cap M$, откуда $\Phi(z) \subset B \cap M$. Далее, если $k \in[0,1]$ и $\rho\left((1-k) z+k z_{0}, M\right)=k R$, то $k \leqslant k(z)$ и $P_{M}\left((1-k) z+k z_{0}\right) \subset \Phi(z)$.

Если $z \in \stackrel{B}{B}\left(z_{0}, R\right) \cap M$, то $k(z)=0$ и $\Phi(z)=\{z\}$, а если $z \in S\left(z_{0}, R\right)$, то $z \in S\left((1-k) z+k z_{0}, k R\right)$ при всех $k \in[0,1]$, и может случиться, что $z \in \Phi(z), \Phi(z) \neq\{z\}$. 
Далее, пусть $z \in B$ и пусть $U$ - открытая окрестность $\Phi(z)$. Рассмотрим последовательность $\left(x_{n}\right)_{n \in \mathbb{N}}$, сходящуюся к $z$. Мы можем выбрать подпоследовательность $\left(z_{n}\right)_{n \in \mathbb{N}}$ таким образом, что последовательность $\left(k\left(z_{n}\right)\right)_{n \in \mathbb{N}}$ является сходящейся к некоторому $k \in[0,1]$. Тогда

$$
(1-k) z+k z_{0}=\lim _{n \rightarrow \infty}\left(1-k\left(z_{n}\right)\right) z_{n}+k\left(z_{n}\right) z_{0}
$$

и, следовательно,

$$
\rho\left((1-k) z+k z_{0}, M\right)=\lim _{n \rightarrow \infty} \rho\left(\left(1-k\left(z_{n}\right)\right) z_{n}+k\left(z_{n}\right) z_{0}, M\right)=\lim _{n \rightarrow \infty} k\left(z_{n}\right) R=k R .
$$

Отсюда $P_{M}\left((1-k) z+k z_{0}\right) \subset \Phi(z) \subset U$. Теперь из полунепрерывности сверху метрической проекции на замкнутые множества в конечномерном пространстве следует, что $\Phi\left(z_{n}\right) \subset U$ при всех достаточно больших $n$, что показывает, что $\Phi$ полунепрерывна сверху. Доказательство леммы 3 завершено.

Следствие. В конечномерном линейном несимметрично нормированном пространстве для замкнутых множеств класс Р-ацикличных множеств совпадает с классом В-ацикличных множеств.

Открытый вопрос. В заключение отметим, что в настоящий момент не решены аналогичные задачи характеризации конечномерных пространств (размерности $\geqslant 3$ ), в которых всякое ограниченное строгое солнце (солнце, связное $\alpha$-солнце) выпукло. Есть основания полагать, что для строгих солнц (и даже солнц) ответ будет аналогичный, приведенному в теореме 1 (конечно, этот так в размерности 2). При этом для положительного решения задачи для строгих солнц не хватает лишь «малого»: показать, что в любом конечномерном пространстве (ограниченное) строгое солнце (солнце) $P$-ациклично. (По поводу связанной с ней давно стоящей задачи о $B$-связности солнц в конечномерных пространствах см., например, [24-26].) В связи со сказанным выше сформулируем гипотезу, что всякое строгое солнце в конечномерном пространстве $B$-стягиваемо и, значит, $B$-ациклично.

Автор выражает благодарность Е. В. Щепину, А. Н. Дранишникову и У. Х. Каримову за консультации.

Работа выполнена при финансовой поддержке РФФИ (проект №13-01-00022).

\section{Библиографический список}

1. Ефимов Н. В., Стечкин С. Б. Некоторые свойства чебышёвских множеств // Докл. АН СССР. 1958. Т. 118, № 1. С. 17-19.

2. Царьков И. Г. Ограниченные чебышёвские множества в конечномерных банаховых пространствах // Матем. заметки. 1984. Т. 36, № 1. С. 73-87.

3. Cobzaş S. Functional analysis in asymmetric normed spaces. Basel : Birkhäuser, 2012.

4. Ахиезер Н. И., Крейн М. Г. О некоторых вопросах теории моментов. Харьков : ОНТИ, 1938.

5. Бородин П. А. О выпуклости $n$-чебышёвских множеств // Изв. РАН. Сер. матем. 2011. Т. 75, № 4. С. 1946. DOI: $10.4213 / \mathrm{im} 4280$.

6. Бердышев В. И. К вопросу о чебышёвских множествах // Докл. АН АзССР. 1966. Т. 22, № 9. С. 3-5.

7. Brøndsted A. Convex sets and Chebyshev sets II // Math. Scand. 1966. Vol. 18. P. 5-15.

8. Brown A. L. Chebyshev sets and the shapes of convex bodies // Methods of Functional Analysis in Approximation Theory : Proc. Intern. Conf., Indian Inst. Techn. Bombay, 16-20.XII.1985 / eds. C. A. Micchelli. Bombay, 1986. P. 97-121.

9. Brown A. L. Chebyshev sets and facial systems of convex sets in finite-dimensional spaces // Proc. Lond. Math. Soc. (3). 1980. Vol. 41. P. 297-339. DOI: 10.1112/ plms/s3-41.2.297.
10. Власов Л. П. Аппроксимативные свойства множеств в линейных нормированных пространствах // УМН. 1973. Т. 28, № 6. С. 3-66.

11. Балаганский В. С., Власов Л. П. Проблема выпуклости чебышёвских множеств // УМН. 1996. Т. 51, № 6. C. 125-188. DOI: 10.4213/rm1020.

12. Царьков И. Г. Компактные и слабо компактные чебышёвские множества в линейных нормированных пространствах // Тр. МИАН СССР. 1989. Т. 189. С. 169184.

13. Алимов A. Р. Всякое ли чебышёвское множество выпукло? // Матем. просвещение. Сер. 3. 1998. № 2. С. $155-172$.

14. Алимов A. Р. О структуре дополнения к чебышёвским множествам // Функц. анализ и его прил. 2001. T. 35, № 3. C. 19-27. DOI: 10.4213/faa255.

15. Bunt L. N. H. Bijdrage tot de theorie der convexe puntverzamelingen. Thesis. Amsterdam : Univ. Groningen, 1934.

16. Карлов М. И., Царьков И. Г. Выпуклость и связность чебышёвских множеств и солнц // Фундам. и прикл. матем. 1997. Т. 3, № 4. С. 967-978.

17. Klee V. L. A characterization of convex sets // Amer. Math. Monthly. 1949. Vol. 56. P. 247-249. DOI: 10.2307/ 2304766.

18. Klee V. L. Convex bodies and periodic homeomor- 
phisms in Hilbert space // Trans. Amer. Math. Soc. 1953. Vol. 74. P. 10-43. DOI: 10.1090/S0002-9947-19530054850-X.

19. Madsen I. B., Milgram R. J. The classifying spaces for surgery and cobordism of manifolds. Princeton, NJ : Princeton Univ. Press, 1979.

20. Dold A. Homology of symmetric products and other functors of complexes // Ann. Math. 1958. Vol. 68, № 1. P. $64-80$. DOI: $10.2307 / 1970043$.

21. Dold A., Thom R. Quasifaserunger und unendliche symmetrische Produkte // Ann. Math. 1958. Vol. 67. P. 239-281. DOI: $10.2307 / 1970005$.

22. Дранищников A. Н. Абсолютные F-значные ретракты и пространства функций в топологии поточечной сходимости // Сиб. матем. журн. 1986. Т. 27, № 3. C. $74-86$.
23. Alimov A. R. A number of connected components of sun's complement // East J. Approx. 1995. Vol. 1, № 4. P. 419-429.

24. Brown A. L. Suns in normed linear spaces which are finite-dimensional // Math. Ann. 1987, Vol. 279. P. 87101. DOI: $10.1007 / \mathrm{BF} 01456192$.

25. Brown A. L. Suns in polyhedral spaces // Seminar of Mathem. Analysis : Proc. / eds. D. G. Álvarez, G. Lopez Acedo, R. V. Caro, Univ. Malaga and Seville (Spain), Sept. 2002 - Feb. 2003. Sevilla : Universidad de Sevilla, 2003. P. 139-146

26. Алимов A. Р. Монотонная линейная связность и солнечность связных по Менгеру множеств в банаховых пространствах // Изв. РАН. Сер. математическая. 2014. T. 78, № 4. C. 3-18. DOI: 10.4213/im8128.

\title{
Convexity of Bounded Chebyshev Sets in Finite-dimensional Asymmetrically Normed Spaces
}

\author{
A. R. Alimov \\ Lomonosov Moscow State University, Faculty of Mechanics and Mathematics, 1, Main Building, Leninskiye Gory, GSP-1, Moscow, \\ 119991, Russia, alexey.alimov@gmail.com \\ The well-known Tsar'kov's characterisation of finite-dimensional Banach spaces in which every bounded Chebyshev set (bounded \\ $P$-acyclic set) is convex is extended to the asymmetrical setting.
}

Key words: bounded Chebyshev set, convexity, asymmetric norm.

This work was supported by the Russian Foundation for Basic Research (project no. 13-01-00022).

\section{References}

1. Efimov N. V., Stechkin S. B. Some properties of Chebyshev sets, Dokl. Akad. Nauk SSSR, 1958, vol.118, no. 1, pp. 17-19 (in Russian).

2. Tsar'kov I. G. Bounded Chebyshev sets in finitedimensional Banach spaces, Math. Notes, 1984, vol. 36, no. 1, pp. 530-537. DOI: 10.1007/BF01139554.

3. Cobzaş S. Functional Analysis in Asymmetric Normed Spaces. Basel, Birkhäuser, 2012.

4. Akhiezer N. I., Krein M. G., O nekotorykh voprosah teorii momentov [Some Problems of the Theory of Moments]. Kharkov, Gonti, 1938.

5. Borodin P. A. On the convexity of $N$-Chebyshev sets. Izv. Math., 2011, vol. 75, no. 5, pp. 889-914. DOI:10.1070/IM2011v075n05ABEH002557.

6. Berdyshev V. I. On a question of Chebyshev sets. Dokl. AN AzSSR 1966, vol. 22, no. 9, pp. 3-5 (in Russian).

7. Brøndsted A. Convex sets and Chebyshev sets II. Math. Scand., 1966, vol. 18, pp. 5-15.

8. Brown A. L. Chebyshev sets and the shapes of convex bodies. Methods of Functional Analysis in Approximation Theory, Proc. Intern. Conf., Indian Inst. Techn. Bombay, 16-20.XII.1985 / eds. C. A. Micchelli. Bombay, 1986, pp. 97-121.

9. Brown A. L. Chebyshev sets and facial systems of convex sets in finite-dimensional spaces. Proc. Lond. Math. Soc. (3), 1980, vol. 41, pp. 297-339. DOI: 10.1112/plms/s3-41.2.297.

10. Vlasov L. P. Approximative properties of sets in normed linear spaces. Russ. Math. Surv., 1973, vol. 28, no. 6, pp. 1-66. DOI:10.1070/RM1973v028n06 ABEH001624.

11. Balaganskii V. S., Vlasov L. P. The problem of convexity of Chebyshev sets. Russ. Math. Surv., 1996, vol. 51, no 6, pp. 125-188. DOI: 10.1070/RM1996 v051n06ABEH003002.

12. Tsar'kov I. G. Compact and weakly compact Chebyshev sets in linear normed spaces. Proc. Steklov Inst. Math., 1990, vol. 189, pp. 199-215.

13. Alimov A. R. Is every Chebyshev set convex? Matematicheskoe prosviashchenie, Ser. 3, 1998, no. 2, pp. 155-172. (in Russian).

14. Alimov A. R. On the structure of the complements of Chebyshev sets. Funct. Anal. Appl., 2001, vol. 35, no. 3, pp. 176-182. DOI: 10.1023/A:1012370610709.

15. Bunt L. N. H. Bijdrage tot de Theorie der Convexe Puntverzamelingen. Thesis. Amsterdam, Univ. Groningen, 1934.

16. Karlov M. I., Tsar'kov I. G., Convexity and connectedness of Chebyshev sets and suns. Fundam. Prikl. Mat., 1997, vol. 3, no. 4, pp. 967-978.

17. Klee V. L. A characterization of convex sets. Amer. Math. Monthly, 1949, vol. 56, pp. 247-249. DOI: 10.2307/ 2304766.

18. Klee V. L. Convex bodies and periodic homeomorphisms in Hilbert space. Trans. Amer. Math. Soc., 
1953, vol. 74, pp. 10-43. DOI: 10.1090/S0002-9947-19530054850-X.

19. Madsen I. B., Milgram R. J. The Classifying Spaces for Surgery and Cobordism of Manifolds. Princeton, NJ, Princeton Univ. Press, 1979.

20. Dold A. Homology of symmetric products and other functors of complexes Ann. Math. (2), 1958, vol. 68, no. 1, pp. 64-80. DOI: $10.2307 / 1970043$.

21. Dold A., Thom R. Quasifaserungen und unendliche symmetrische Produkte. Ann. Math. (2), 1958, vol. 67, no. 2, pp. 239-281. DOI: $10.2307 / 1970005$

22. Dranishnikov A. N. Absolute F-valued retracts and spaces of functions in the topology of pointwise convergence. Siberian Math. J., 1986, vol. 27, no. 3, pp. 366-376. DOI: 10.1007/BF00969273.
23. Alimov A. R. A number of connected components of sun's complement East J. Approx., 1995, vol. 1, no. 4, pp. 419-429.

24. Brown A. L. Suns in normed linear spaces which are finite-dimensional Math. Ann., 1987, vol. 279, pp. 87-101. DOI: $10.1007 /$ BF01456192.

25. Brown A. L. Suns in polyhedral spaces. Seminar Math. Anal., Proceedings / eds.: D. G. Álvarez, G. Lopez Acedo, R. V. Caro, Univ. Malaga and Seville (Spain), Sept. 2002 - Feb. 2003. Sevilla, Universidad de Sevilla, 2003, pp. 139-146.

26. Alimov A. R. Monotone path-connectedness and solarity of Menger-connected sets in Banach spaces. Izv. Math., 2014, vol. 78, no. 4, pp. 641-655. DOI: 10.1070/IM2014v078n04ABEH002702.

УДК 517.518

\title{
О РАСХОДИМОСТИ ПОЧТИ ВСЮДУ РЯДОВ ФУРЬЕ НЕПРЕРЫВНЫХ ФУНКЦИЙ ДВУХ ПЕРЕМЕННЫХ
}

\begin{abstract}
Н. Ю. Антонов
Доктор фризико-математических наук, заведующий отделом теории приближения фрункций, Институт математики и механики им. Н. Н. Красовского УрО РАН, Екатеринбург, Nikolai.Antonov@imm.uran.ru

Рассматривается один вид сходимости двойных тригонометрических рядов Фурье, промежуточный между сходимостью по квадратам и $\lambda$-сходимостью при $\lambda>1$. Построен пример непрерывной функции двух переменных, ряд Фурье которой расходится в указанном смысле почти всюду.
\end{abstract}

Ключевые слова: кратные ряды Фурье, сходимость почти всюду.

Пусть $\mathbb{N}, \mathbb{Z}, \mathbb{R}$ - множества натуральных, целых и действительных чисел соответственно, $\mathbb{T}^{2}=[0,2 \pi)^{2}-$ двумерный тор,

$$
\sum_{(k, l) \in \mathbb{Z}^{2}} a_{k, l} e^{i(k x+l y)}, \quad(x, y) \in \mathbb{T}^{2},
$$

- двойной тригонометрический ряд Фурье $2 \pi$-периодической по каждой переменной и суммируемой на $\mathbb{T}^{2}$ функции $f$ и для $(m, n) \in \mathbb{N}^{2}$

$$
S_{m, n}(f, x, y)=\sum_{\substack{(k, l) \in \mathbb{Z}^{2}: \\|k| \leqslant m,|l| \leqslant n}} a_{k, l} e^{i(k x+l y)}
$$

- $(m, n)$-я прямоугольная частичная сумма ряда (1).

Говорят, что ряд (1) сходится по Прингсхейму в точке $(x, y) \in \mathbb{T}^{2}$, если существует

$$
\lim _{\min \{m, n\} \rightarrow \infty} S_{m, n}(f, x, y) .
$$

Пусть $\lambda \geqslant 1$. Ряд (1) называется $\lambda$-сходящимся в точке $(x, y) \in \mathbb{T}^{2}$, если существует предел (2), рассматриваемый только по тем парам $(m, n)$, для которых $1 / \lambda \leqslant m / n \leqslant \lambda$. В случае $\lambda=1 \lambda$-сходимость называется сходимостью по квадратам.

Аналогичным образом определяются кратный ряд Фурье, его прямоугольные частичные суммы и упомянутые виды сходимости для функций $d$ переменных при $d>2$.

Через $f \in C\left(\mathbb{T}^{2}\right)$ и $L^{p}\left(\mathbb{T}^{2}\right), p \geqslant 1$, будем обозначать множества $2 \pi$-периодических по каждой переменной функций, которые непрерывны и суммируемы с $p$-й степенью соответственно.

В случае сходимости по квадратам (кубам) известно $[1,2]$, что если функция $f$ принадлежит классу $L^{p}\left(\mathbb{T}^{d}\right), p>1, d \geqslant 2$, в частности, если $f$ непрерывна, то ряд Фурье этой функции сходится (по 